\title{
Haemangiopericytic meningioma of the sacral canal: a case report
}

\author{
L R BRIDGES, * S ROCHE, $\uparrow$ L NASHEF, $\dagger$ F CLIFFORD ROSE $\dagger$ \\ From the Departments of Histopathology* and Neurology, $\uparrow$ Charing Cross Hospital, London, UK
}

SUMMARY A case of haemangiopericytic meningioma of the sacral canal in a 25 year old man, an uncommon tumour at a rare site, is described. The tumour was malignant and largely undifferentiated although there was light and electron microscopic evidence of dual differentiation in areas towards haemangiopericytoma and meningioma. The patient, with cauda equina syndrome, was treated by partial resection and post-operative radiotherapy and remains well 12 months after treatment.

Haemangiopericytic meningiomas represent $1-2 \%$ of meningeal tumours; although 30 intraspinal cases were described in a recent review, ${ }^{1}$ only one example involving the sacral canal has been previously reported. ${ }^{2}$ For mainly historical reasons the tumours have several names (haemangiopericytoma, angioblastic meningioma, meningeal sarcoma) although their histogenesis is in fact unknown. ${ }^{3}$ Microscopically they are mesenchymal tumours which may be undifferentiated or show partial differentiation towards haemangiopericytoma and meningioma. ${ }^{4}$ Their distinction from typical meningioma is important because of their often aggressive biological behaviour.

\section{Case report}

Clinical features

A 25 year old airport porter presented with a 10 week history of low back pain radiating to the right buttock, posterior leg and heel. This was worse at night, on bending his neck, laughing or sneezing. When first seen he had constant pain requiring regular analgesia, right buttock and posterior leg numbness; he was constipated and complaining of hesitancy before and dribbling after micturition. On examination there was limitation of lumbar spine movement with straight leg

Address for reprint requests: Dr L R Bridges, Department of Histopathology, Charing Cross Hospital, Fulham Palace Road, London W68RF, UK.

Received 13. March 1987 and in revised form 30 June 1987. Accepted 24 August 1987 raising limited to $20^{\circ}$ on the right and $30^{\circ}$ on the left. Muscle power was normal. The right ankle tendon reflex was absent. The anal reflex was present but anal tone was reduced. Lightī touch and pin prick sensation were reduced over the right buttock, scrotum and posterior thigh. Lumbo-sacral radi culography showed a right-sided sacral extradural mass displacing and compressing the theca and nerve roots. Ches radiograph, full blood count, erythrocyte sedimentation rate, calcium, liver function tests, urea, electrolytes, urine and serum protein electrophoresis, carcino-embryonic antigen and alpha-foetoprotein levels were normal. Computed tomography (CT) showed a soft-tissue tumour of the sacral canal beginning at the $\mathrm{S} 1$ vertebra. There was destruction of S1-3 vertebral laminae and the right sacro-iliac joint and extension into the right buttock (fig 1). An isotope bone scan showed increased uptake confined to this region. Surgical exploration of the sacral canal revealed a friable, soft, vascular extradural tumour requiring active haemostasis. Subtotal resection was performed. After operation the patient received radiotherapy to the sacrum totalling 5,040 centiGrays (cGY) in 28 fractions over 48 days. Nine months after the operation he returned to work and remains well apart from slight right buttock numbness, residual limitation of straight leg raising bilaterally to $60^{\circ}$ and an absent right ankle jerk.

\section{Histology}

The surgical specimens comprised brown, fleshy fragments measuring a total $4.0 \times 3.0 \times 2.0 \mathrm{~cm}$. Histology of these showed a homogeneous, highly cellular malignant neoplasm composed of oval and spindle-shaped cells arranged in a solid pattern around capillaries and dilated blood-filled sinusoids (fig 2). Occasional cellular whorls were found (fig $3)$, usually surrounding a small blood vessel. Nuclei were clear, measured between one and three red cell diameters 


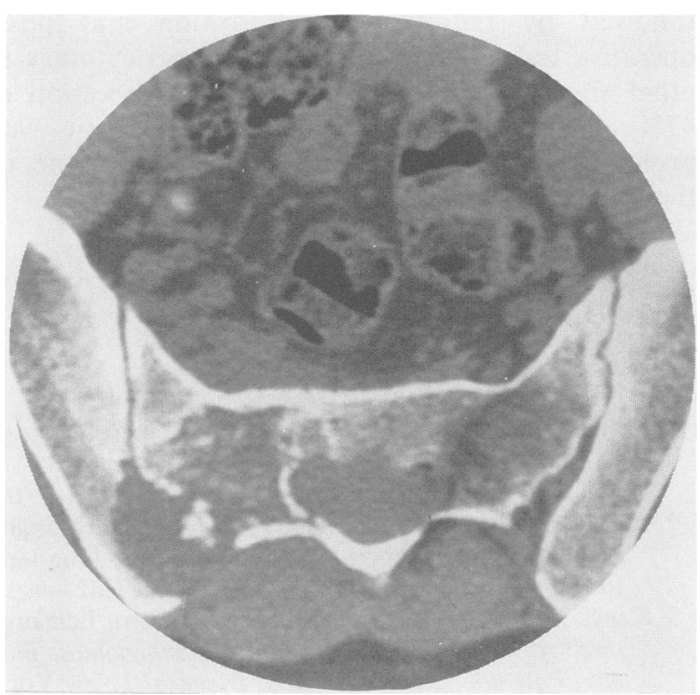

Fig 1 CT scan showing soft tissue tumour in sacral canal eroding sacral bone and extending into right buttock.

and were round or oval and angulated in shape. Cytoplasm was pale with indistinct borders. The vascular channels were lined by flattened endothelial cells with elongated, uniform dark nuclei. In some areas reticulin fibres demonstrated by James silver method were abundant and surrounded the vessels and individual tumour cells. In other areas pericellular reticulin was sparse. The cells of the whorls were individually outlined by reticulin. Collagen deposition was present

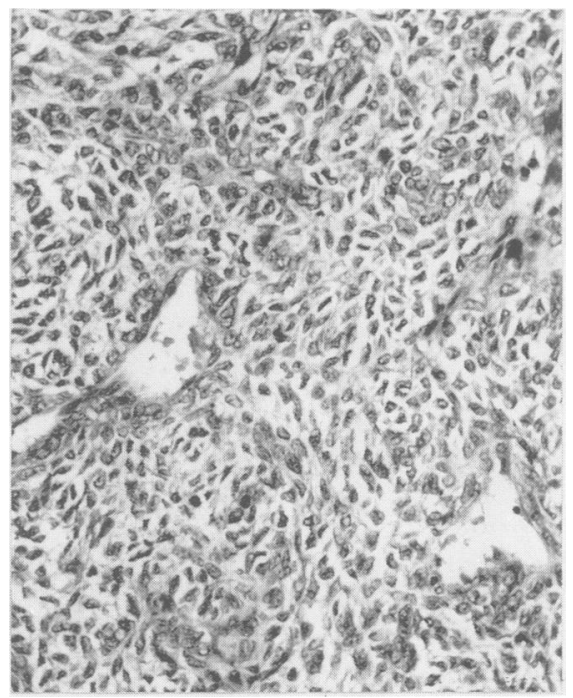

Fig 2 Densely cellular diffuse neoplasm arranged around prominent sinusoids resembling haemangiopericytoma (haematoxylin and eosin $\times 170$ ).

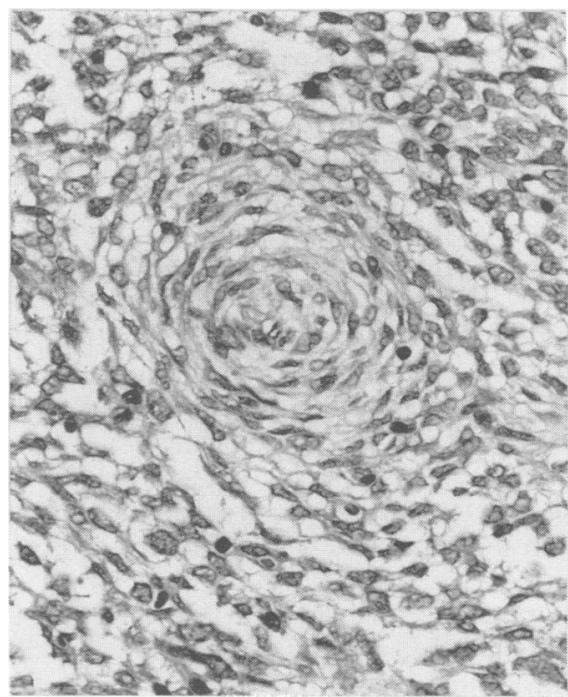

Fig 3 Focal whorl formation within sheets of densely packed cells suggestive of transition to typical meningioma (haematoxylin and eosin $\times 170$ ).

around several large vessels. A few cells contained coarse brown granules of lipofuscin. Throughout the tumour there was nuclear pyknosis and karyorrhexis. Mitotic figures averaged 9 per 10 high power fields. There were frequent areas of recent haemorrhage. The immunoperoxidase method was positive in tumour cells for vimentin intermediate filament and negative for factor VIII/von Willebrand related antigen, cytokeratin intermediate filament CAM 5·2, S100-protein and epithelial membrane antigen. Endothelial cells stained positively for factor VIII and vimentin.

Fresh tissue was placed in Karnovsky fixative prepared by conventional methods for electron microscopy. This showed closely-packed cells with elaborately intertwined, elongated cytoplasmic processes. There were occasional intermediate junctions but no desmosomes. Nuclei contained plentiful euchromatin, internal reticular wisps and subnucleolemmal condensations of heterochromatin and one or two peripheral small nucleoli. The cytoplasm was lucent with few organelles including areas of rough endoplasmic reticulum, free ribosomes, aggregates of mitochondria and loose networks of fine filaments. Focal deposits of external laminallike material were present on the outer surface of tumour cells. The stroma contained small amounts of collagen and ground substance, extravasated red cells, macrophages and normal capillaries. These findings suggest a malignant haemangiopericytic meningioma.

\section{Discussion}

Microscopically there was a predominantly solid pattern of cells with areas resembling haemangiopericytoma (fig 2) and areas of sparse reticulin and whorl formation (fig 3) previously considered "transitional" to typical meningioma. ${ }^{4}$ The immu- 
noperoxidase reaction for $\mathrm{S}-100$ protein was negative as in seven previous cases of haemangiopericytic meningioma ${ }^{5}$ and unlike 13 previous cases of typical meningioma where the reaction was positive. ${ }^{6}$ Ultrastructurally the cells were poorly differentiated and without desmosomes, a typical feature of meningiomas; however, there were elongated and intertwined cytoplasmic processes, previously held as evidence of transition to typical meningioma. ${ }^{4}$ The ultrastructural features were not those of mature pericytes which have abundant external lamina and cytoplasmic filaments. ${ }^{4}$ Thus, we are unable to provide evidence of either pericytic or meningothelial origin. The dual haemangiopericytomatous and meningiomatous differentiation are consistent with origin from a primitive, connective tissue stem cell as postulated by Horten et al. ${ }^{4}$

This patient presented with a cauda equina syndrome as did the previously reported case. ${ }^{2}$ Patients with intraspinal haemangiopericytic meningiomas at other levels usually present with spinal cord compression or with radicular pain. Usually, as in this case, there is gradual progression over several months. Both sexes are equally affected and the average age of presentation at 32 years is 18 years younger than for typical meningioma. ${ }^{1}$ At surgery the tumours are typically extradural, unencapsulated, and extend into the mediastinum, retroperitoneum, pelvis or buttock via eroded bone or intervertebral foramina. For these reasons surgical excision is usually incomplete. Although haemangiopericytomas may be clearly cytologically benign or malignant there are cases with borderline appearances in which biological behaviour may be impossible to predict. ${ }^{7}$ In the present tumour there was clear evidence of malignancy with a high mitotic rate, necrosis and local invasion. Treatment was by maximal resection followed by radiotherapy. Resection and postoperative radiotherapy of haemangiopericytomas at other sites have resulted in complete regression in $47 \%$ of cases. ${ }^{8}$ Although our patient remains well prolonged follow-up is required because some of these tumours have later metastasised. ${ }^{12}$

\section{References}

1 Muller JP, Destee A, Verier A, et al. Les hemangiopericytomes intrarachidiens: deux observations et revue de la literature. Neurochirurgie 1986;32:140-6.

2 McMaster MJ, Soule EH, Ivins JC. Haemangiopericytoma: a clinicopathological study and long-term follow-up of 60 patients. Cancer 1975;36:2232-44.

3 Kepes JJ. Hemangiopericytic meningiomas or hemangiopericytomas of the meninges. In: Meningiomas: Biology, Pathology and Differential Diagnosis. New York: Masson, 1982:87-90.

4 Horten BC, Urich H, Rubinstein LJ, Montague SR. The angioblastic meningioma: a reappraisal of a nosological problem. J Neurol Sci 1977;31:387-410.

5 Nakamura M, Inoue HK, Ono N, Kunimine H, Tamadad N J. Analysis of haemangiopericytic meningiomas $b \bar{\phi}$ immunochemistry, electron microscopy and cell cuf ture. J Neuropath Exp Neurol 1987;46:57-71.

6 Theaker JM, Gatter KC, Esiri MM, Fleming KA. Epi̊̊ $\vec{~}$ thelial membrane antigen and cytokeratin expressio $\bar{D}$ by meningiomas: an immunohistological study. J Clin Pathol 1986;39:435-9.

7 Enzinger FM, Weiss SW. Haemangiopericytoma. In Soft Tissue Tumours. St Louis: CV Mosby, 1986 463-81.

8 Mira JG, Chu FCH, Fortner JG. The role of radiotherapy in the management of malignant haemangiopericytoma: report of 11 new cases and review of the literature. Cancer 1977;39:1254-9. 